Association for Information Systems AIS Electronic Library (AISeL)

Wirtschaftsinformatik Proceedings 2003

Wirtschaftsinformatik

September 2003

\title{
Netzeffekte im E-Business
}

\author{
Wolfgang König \\ Johann Wolfgang Goethe-Universität Frankfurt am Main, koenig@wiwi.uni-frankfurt.de \\ Tim Weitzel \\ Johann Wolfgang Goethe-Universität Frankfurt am Main
}

Follow this and additional works at: http://aisel.aisnet.org/wi2003

\section{Recommended Citation}

König, Wolfgang and Weitzel, Tim, "Netzeffekte im E-Business" (2003). Wirtschaftsinformatik Proceedings 2003. 2.

http://aisel.aisnet.org/wi2003/2

This material is brought to you by the Wirtschaftsinformatik at AIS Electronic Library (AISeL). It has been accepted for inclusion in Wirtschaftsinformatik Proceedings 2003 by an authorized administrator of AIS Electronic Library (AISeL). For more information, please contact elibrary@aisnet.org. 
In: Uhr, Wolfgang, Esswein, Werner \& Schoop, Eric (Hg.) 2003. Wirtschaftsinformatik 2003: Medien - Märkte - Mobilität, 2 Bde. Heidelberg: Physica-Verlag

ISBN: 3-7908-0111-9 (Band 1)

ISBN: 3-7908-0116-X (Band 2)

(C) Physica-Verlag Heidelberg 2003 


\title{
Netzeffekte im E-Business
}

\author{
Wolfgang König, Tim Weitzel \\ Johann Wolfgang Goethe-Universität Frankfurt am Main
}

Zusammenfassung: Eine grundlegende Fragestellung im E-Business liegt in der geeigneten Vernetzung verschiedener Akteure. Dabei gibt es netzwerkspezifische Koordinationsprobleme, die aus der Existenz von Netzeffekten resultieren. Gegenstand dieses Beitrages ist eine ökonomische Analyse der Existenz und Effizienz. von Gleichgewichten in Kooperationsnetzen. Computersimulationen zeigen vielversprechende Resultate bezüglich der prinzipiellen Lösbarkeit „klassischer“ Netzwerkprobleme wie insbesondere des Start-up-Problems.

Schlüsselworte: Netzeffekt, E-Business, Gleichgewicht, Standard

\section{Einleitung}

"If the dominant symbol of the industrial economy is a factory, then the emblem of the modern economy is a network."

$\left[\mathrm{Maub}^{+} 00\right]$

Das 20. Jahrhundert war gekennzeichnet durch eine steigende Bedeutung nachfrageseitiger Skaleneffekte, die in sehr unterschiedlichen Kontexten als positive Netzeffekte diskutiert werden. Die Bedeutung von Netzeffekten resultiert in vielen Fällen aus Kompatibilitätserfordernissen. Daher spielen Netzeffekte eine zentrale Rolle als Wertbeitrag im E-Business und werden großenteils in der Literatur über Standards untersucht [Weit03]. Nach einer allgemeinen Definition ist Electronic Business jede geschäftliche Transaktion, deren Teilnehmer elektronisch interagieren. Im Gegensatz zu Electronic Commerce, bei welchem der Endkundenkontakt (Business-to-Consumer, B2C) im Vordergrund steht, handelt es sich dabei um die digitale Abwicklung von Geschäftsprozessen (in und) zwischen Unternehmen [Weit ${ }^{+} 01$; Schi00]. Eine grundlegende Fragestellung des E-Business liegt entsprechend in der geeigneten Vernetzung verschiedener Akteure und Systeme mit dem Ziel, Netzeffekte zu realisieren.

Während bis Mitte des letzten Jahrhunderts, getrieben durch den Ersten Weltkrieg, der Schwerpunkt der meisten Standardisierungsaktivitäten auf produktionsseitiger Vereinheitlichung lag (Reduktion von Produkt- und Prozessvielfalt), wandelte sich der Fokus später in Richtung Kompatibilitätsstandards. Brian Arthur nennt dies 
den Wechsel von "Alfred Marshall's World" zu einer "Increasing-Returns World" [Arth96]. Da Standards Kompatibilität ermöglichen und damit die Grundlage zur Nutzung vieler Synergien sind [Gabe87; Gabe91; FaSa87; Nigg94; Klei93], spricht man davon, dass Standards Netzwerke von Nutzern konstituieren [BeFa94; DaGr90]. Prominente Beispiele aus der Standardisierungsliteratur sind Schienenbreite bei Eisenbahnen, Schraubendurchmesser, Kernkraftwerke, Tatstaturanordnungen, VCR-Systeme, Web-Browser, SSO-Software, EDI-Netze und OfficeSoftware [WeWe02; Weit03]. Dabei entsteht ein Netzeffekt im Eisenbahnbeispiel durch die Normung von Schienenbreite als Grundlage der Verwendung fremder Schienennetze [Kind83, S.384-385] und das Schraubenbeispiel beschreibt in ähnlicher Weise das Zusammenpassen (Interoperabilität) von Schrauben und Schraubenziehern. Andere Netzeffekte finden sich zwischen elektrischen Geräten und Stromspannungen, Kraftfahrzeugen und Betriebsmitteln oder Videorekordern und Kassetten [Plum37, S.21; WaWa83, S.125; Heme75, S.3; GeHö95, S.21-29; Bund98]. Weitere bedeutende Beispiele wie EDI-Netze zum automatisierten Geschäftsdatenaustausch und Verzeichnisdienste fokussieren mehr auf den direkten Informationsaustausch, während die Beispiele aus dem Bereich von Kernreaktoren (Leichtwasser- vs. Schwerwasser- vs. Gas-Graphitreaktoren) [Cowa90, S.559560] stärker den Lernkurveneffekt der Technologieentwicklung als Grundlage haben. Habermeier [Habe89; Habe94] unterscheidet zwischen Effekten auf Grund von Netzwerkteilnahme ("joining a well-established network"), informationalen Skaleneffekten (z. B. Markenbekanntheit), Komplementäreffekten (Videorekorder und Kassette) und dynamischen Lerneffekten (je mehr eine Technologie genutzt wird, desto stärker wird sie optimiert und ihre Vorteile werden erkennbar).

In diesem Beitrag sollen die als direkte Netzeffekte [KaSh85, S.424] bekannt gewordenen Interoperabilitätsvorteile aus Netzwerkteilnahme untersucht werden, die insbesondere im E-Business-Kontext durch IuK-gestützte Vernetzung Kooperationsvorteile ermöglichen. Wie in EDI-Netzen und auch betrieblichen Intranets können so Partner "participate in networks that allow them to share databases, have access to large selections of compatible software, exchange documents (...) or simply communicate directly" [BeFa94, S.117].

Auch wenn die Metapher Netzwerk zur Beschreibung unterschiedlichster vorteilhafter Strukturen zwischen Akteuren in Theorie wie Praxis weit verbreitet ist, gibt es übergreifende, netzwerkspezifische Koordinationsprobleme, die aus der Existenz von Netzeffekten resultieren und möglicherweise neue Koordinationskonzepte im E-Business erfordern. Gegenstand diese Beitrages ist eine ökonomische Analyse der Existenz und Effizienz von Gleichgewichten in den beschriebenen Kooperationsnetzen bei unterschiedlichen grundsätzlichen Koordinationsrahmenbedingungen (zentrale versus dezentrale Steuerung). Hierzu wird in Kapitel 2 ein Überblick über die wichtigsten Erkenntnisse in der Netzeffektliteratur gegeben und diese in Kapitel 3 zu einem Forschungsrahmen für Kooperationsnetze, wie sie typischerweise in E-Business-Kontexten zu finden sind, weiterentwickelt. Hierauf aufbauend zeigt eine Computersimulation in Kapitel 4 vielversprechende Resulta- 
te bezüglich der prinzipiellen Lösbarkeit „klassischer“ Netzwerkprobleme wie insbesondere des Start-up-Problems. Ein zentrales Ergebnis besteht darin, dass häufig eine Vielzahl individueller Netzwerkakteure bei zentraler Koordination am besten gestellt ist und dass grundlegende Koordinationsprobleme im E-Business durch vergleichsweise einfache Lösungsansätze adressiert werden können.

\section{Netzeffekte als theoretische Grundlage}

\subsection{Netzeffekte als Externalitäten}

Für viele Märkte gilt, dass Kaufentscheidungen eines Konsumenten die Entscheidungen anderer beeinflussen. In der klassischen ökonomischen Literatur werden derartige Interdependenzen wie Bandwagon-, Snob- und Veblen-Effekte ausführlich diskutiert [Leib50; CeKa82]. Darüber hinaus unterliegen bestimmte Märkte sog. Netzeffekten oder nachfrageseitigen Skalenerträgen. Dies impliziert einen positiven Zusammenhang zwischen der Bereitschaft, Netzeffektgüter zu adoptieren, und der Anzahl sonstiger Nutzer dieses Gutes. Prominente Beispiele sind Märkte für IuK-Technologien oder auch das Telefon. ${ }^{1}$ Der Nutzen, ein Telefon zu besitzen, ist abhängig von der Anzahl der Personen, die hiermit erreicht werden können. Im Zuge der rasanten Entwicklung und wachsenden Bedeutung von IuKTechnologien in den letzten Jahren entstand eine eigene Forschungsrichtung mit dem Ziel, die mit positiven Netzeffekten einhergehenden Phänomene zu erklären und ihre Implikationen für (marktliche) Koordination und Effizienz zu untersuchen. Die ersten richtungsweisenden Beiträge zu diesem vergleichsweise jungen Forschungsgebiet, das nachfolgend als Theorie der positiven Netzeffekte bezeichnet wird, stammen aus der ersten Hälfte der achtziger Jahre und untersuchen Standards aus dem Blickwinkel der Diffusion technologischer Innovationen.

Die zentrale Problematik besteht darin, dass Netzeffekte als positive, konsumseitige Externalitäten pareto-inferiore Marktergebnisse implizieren können. Katz und Shapiro [KaSh85] führen eine Unterscheidung zwischen direkten und indirekten

1 Die wettbewerbliche Bedeutung von Standards darin, dass Standards konstituierendes Element von Netzen sind und somit notwendige Voraussetzung der Realisierung der hiermit verbundenen Nutzenpotentiale. Daher ist Kompatibilität auch eine Aktionsvariable der Hersteller von Netzeffekten unterliegenden Produkten, da sie Entscheidungen über das Ausmaß der Kompatibilität mit anderen Produkten womöglich konkurrierender Anbieter treffen müssen. Hohe Kompatibilität zu Konkurrenzprodukten bzw. zu gleichen Standards komplementäre Produkte erlauben das Nutzen fremder installed bases. Allgemein können Standards daher Wettbewerb von einem intertechnologischen zu einem intratechnologischen Kampf verlagern. 
Netzeffekten ein. Direkte Netzeffekte beschreiben demnach einen direkten $\mathrm{Zu}$ sammenhang zwischen Nutzerzahl und Wert eines Netzeffektgutes (,direct physical effect" [KaSh85, S.424]), wie dies beim Telefon der Fall ist. Dagegen resultieren indirekte Netzeffekte aus Interdependenzen im Konsum komplementärer Güter, Beispiele sind das Beratungsangebot im Umfeld von Standardsoftware oder das Zusammenspiel von Betriebssystemen und Anwendungssoftware. Da Kompatibilität bzw. Standardisierung allgemein nur im Zusammenhang mit anderen gesehen werden kann, sind Märkte, auf denen Kompatibilität eine wichtige Produkteigenschaft ist, stets auch Märkte, auf denen starke Netzeffekte zu finden sind. Kindleberger [Kind83] beschreibt aus dem Öffentlichen-Gut-Charakter von Standards (Netzwerkexternalitäten) resultierende Free-rider-Probleme, die sich z. B. aus der Schwierigkeit ergeben, wer die Kosten von Standardisierungsbemühungen tragen soll, wenn die Ergebnisse durch alle frei nutzbar sind. Arthur [Arth83; Arth89] zeigt, dass Diffusionsprozesse von Produkten, die ,increasing returns“, also Netzeffekten bzw. steigenden Skalenerträgen in der Nutzung unterliegen, mehrere Gleichgewichte aufweisen, die jeweils dadurch gekennzeichnet sind, dass eines der alternativen Produkte letztendlich monopolistisch den Markt beherrscht („Lock-in“). Da im Falle nichtproprietärer Standards der Diffusionsprozess von kleinen, zufälligen Ereignissen sehr früher Diffusionsphasen bestimmt werden kann und damit nicht ergodisch (und damit ,pfadabhängig“) ist, kann das letztendliche Marktergebnis nicht vorhergesagt werden. Als Beispiel wird häufig der Kampf um die Videorekorderformate VHS versus Beta oder auch zwischen verschiedenen Automotoren angeführt [Weit03]. Analog zu diesen Aussagen zeigen Besen und Farrell [BeFa94], dass viele Netzwerke instabil sind. Hiernach können auf Netzwerkmärkten tendenziell nur selten mehrere inkompatible Technologien koexistieren ('tippy networks'), wie derzeit etwa der Internet Explorer den ehemaligen Marktführer Netscape Navigator verdrängen konnte.

Die Argumentation in der traditionellen Netzeffektliteratur folgt durchweg einem ähnlichen Pfad: Die Diskrepanz zwischen privatem und kollektivem Nutzen bezüglich der Auswahl eines Standards führt bei Existenz von Netzeffekten möglicherweise zu pareto-dominierten Marktergebnissen, also Marktversagen in dem Sinne, dass Netzeffekte die Gefahr der falschen Technologieadoption (zu viele Technologien, zu wenige Technologien, falsche Technologien) bergen. Insgesamt kann es aus Sicht eines Gesamtnetzes sowohl zu einer Über- als auch zu einer Unterversorgung mit Standards kommen. Eine Unterversorgung (excess inertia) mag dadurch entstehen, dass keiner das überproportionale Risiko einer frühen Auswahl treffen möchte, um nicht der Gefahr ausgesetzt zu sein, in einem schließlich zu kleinen Netz zu ,stranden“ und Wechsel- oder Opportunitätskosten tragen zu müssen, sollte die Mehrzahl späterer Netzteilnehmer sich für eine andere Technologie entscheiden. Unter nicht vollständiger Information kann dieses Start-up-Problem die Einführung verhindern, selbst im Falle homogener Präferenzen der entscheidenden Akteure für eine Standardisierung. In der betrieblichen Praxis wird dieses Phänomen auch als ,aggressives Abwarten“ beschrieben und zeigt sich beispielsweise im „Aussitzen“ von Versionswechseln in vielen Abteilungen eines um 
Standardisierung bemühten Konzerns [WeSK01]. Im Gegensatz dazu zeigen Katz und Shapiro [KaSh86], dass es in ,sponsored networks“ (einzelne Akteure halten Rechte und können andere von der Nutzung ausschließen) auch zu einer Überversorgung (excess momentum) des Marktes durch Preissetzung für den Standard seitens des marktmächtigen Anbieters kommen kann, indem dieser frühe Käufer subventioniert und die Rente späterer Konsumenten der dann wertvolleren Technologie abschöpft. Im Falle vollständiger Information und symmetrischer Präferenzen bezüglich der Vorteilhaftigkeit von Standards löst ein Bandwagon-Prozess das Koordinationsproblem, indem frühe Nutzer mit vergleichsweise hohem Standalone-Nutzen einen Lawinen- oder Dominoeffekt starten. Nichtsdestoweniger zeigen Farrel und Saloner [FaSa86], dass auf Grund strategischen Verhaltens nicht einmal perfekte Kommunikation Excess-inertia- oder -momentum-Probleme zuverlässig lösen kann. Im Falle proprietärer Netzwerke besteht zwar prinzipiell die Möglichkeit, die Netzeffekte zu internalisieren, also die Fernwirkungen explizit zu berücksichtigen. So existieren Anreize für die Besitzer der jeweiligen Technologien, z. B. durch intertemporale Preisstrategien Start-up-Probleme zu lösen. Problematisch bleibt hier jedoch die nicht zwingende Erreichung eines sozialen Optimums im Sinne eines auf neoklassischen Märkten zu erwartenden paretoeffizienten Optimums [Weit ${ }^{+}$03].

Verschiedene methodische Ansätze stellen die Netzeffektdiskussion auf eine vergleichsweise breite, allerdings bislang keineswegs einheitliche oder abgeschlossene Basis. Kleinemeyer [Klme98] und Yang [Yang97] unterscheiden verschiedene Perspektiven der Betrachtung von Netzeffekten. Empirische Ansätze versuchen, die Existenz und Höhe von Netzeffekten durch Regressionsanalysen nachzuweisen und hedonische Preisfunktionen für Netzeffektgüter zu schätzen [HaTe90; Gand94; EcHi95; Moch95; Gröh99]. Theoretische Arbeiten bestehen meist aus Gleichgewichtsanalysen zur Untersuchung von Start-up-Phänomenen [Rohl74; OrSm81; KaSh85; KaSh94; Wies90; BeFa94; EcHi95], Marktversagen [FaSa85; FaSa86; KaSh86; KaSh92; KaSh94; Gröh99], Instabilitäten in Netzeffektmärkten ("tippy networks") [Arth89; Arth96; BeFa94; FaSa85; KaSh94; ShVa98] sowie Pfadabhängigkeiten und nicht-ergodischen Diffusionsprozessen [Davi85; Arth89; BeFa94; KaSh94; LiMa95b].

\subsection{Zusammenfassung der wichtigsten Aussagen der traditionellen Netzeffekttheorie}

Eine ausführliche Übersicht sowie verwandte Literatur aus angrenzenden Bereichen (etwa Actor Network Theory, Infrastrukturtheorien, Herstellerstrategien oder wettbewerbliche Implikationen) bieten [Weit03; Weit ${ }^{+} 00$ ], eine reichhaltige Online-Bibliographie [Econ00; Agre98]. Zusammenfassend sind dies die wichtigsten Ergebnisse der traditionellen Netzeffekt- und Diffusionstheorie: 
- Netzeffekte implizieren multiple Gleichgewichte. Ein Lock-in des Marktes in eine (Monopol-)Technologie ist zu erwarten. Häufig sind diese Marktlösungen pareto-inferior (vulgo Marktversagen) [Arth89; KaSh85; KaSh86; Thum95].

- Netzmärkte (Softwaremärkte) sind typischerweise instabil, das heißt, dass rivalisierende Technologien selten längerfristig koexisitieren und dass der Umschwung zugunsten eines Gewinners sehr plötzlich erfolgen kann [BeFa94, S.118; ShVa98, S.176].

- Ein Start-up-Problem verhindert den Wechsel auf überlegene Technologien; excess inertia (Unterstandardisierung) resultiert aus der Tatsache, dass keiner das überproportionale Risiko der ersten Technologieeinführung eingehen möchte [FaSa85; FaSa86].

- Ebenso kann es zu Überstandardisierung (excess momentum) kommen, wenn beispielsweise durch intertemporale Preisstrategien (z. B. niedrige Einstiegspreise der anbietenden Firmen) rasch eine kritische Nutzermasse aufgebaut werden soll [ $\mathrm{FaSa} 86]$.

\subsection{Kritik an der traditionellen Netzeffekttheorie}

Zu einer ausführlichen Kritik der traditionellen Netzeffekttheorie siehe [LiMa94] [Weit $\left.{ }^{+} 00\right]$ [Weit03]. Zu den wichtigsten Kritikpunkten gehören die folgenden:

- Ungenaue Unterscheidung von direkten und indirekten Netzeffekten trotz unterschiedlicher ökonomischer Implikationen [KaSh94; LiMa94].

- Empirische Defizite beim Nachweis von Marktversagen; allgemeiner: Schwierigkeiten einer sinnvollen Definition von „heilbarem Marktversagen“ bei unvollständiger Information und rationalen Akteuren (eine gängige Annahme in Netzeffektmodellen) [LiMa90].

- Unrealistische Annahme kontinuierlich steigender, homogener Netzeffekte und Vernachlässigung von steigenden Netzeintrittskosten [LiMa95].

- Fehlende Unterscheidung zentral und dezentral koordinierter Netze bzw. der institutionellen Einbettung der Akteure; fehlende normative Kraft der Modelle, insbesondere keine dezentralen Lösungsansätze bzw. Entscheidungshilfen für Nutzer von Netzeffektgütern; Vernachlässigung des Einflusses der individuellen Netzwerkumgebung auf die Übernahmeentscheidung [Weit ${ }^{+} 00$; West03].

Neben einer reduzierten Erklärungskraft traditioneller Ansätze machen diese offenen Probleme eine Übertragung der Ergebnisse auf realwirtschaftliche Entscheidungsprobleme individueller Akteure, etwa bei der Gestaltung elektronischer Märkte, dem Aufbau und der Koordination von Wertschöpfungsnetzwerken oder firmeneigenen Intranets, schwierig. Insbesondere interdisziplinäre Ansätze, die Erkenntnisse aus Disziplinen wie der Soziologie, Geographie oder Medizin in die 
Netzwerkforschung einzubringen und für E-Businessfragen zugänglich zu machen suchen, können vielversprechende Wege aufzeigen, die Erklär- und Gestaltungskraft einer zukünftigen, umfassenden Theorie der Netzwerke zu erhöhen [We$\mathrm{Kö03].}$

\section{Ein Forschungsrahmen für Koordinationsprobleme in Netzwerken}

Unsere Grundhypothese ist, dass die Asymmetrie zwischen individuellen und kollektiven Netzwerkteilnahmeanreizen die automatische effiziente Lösungsfindung, die man auf einem neoklassischen Markt erwartet, zerstört. Aus theoretischer Sicht ist dies nicht überraschend, da Netzeffekte als Externalität die zwangsläufige Transmission von lokaler zu globaler Effizienz verhindern. In diesem Abschnitt werden wichtige Grundlagen der Netzeffekttheorie (Netzeffekte und ihre Auswirkungen) und der traditionellen Ökonomie (Externalitäten, Existenz und Effizienz von Gleichgewichten, neoklassische Wohlfahrtsmechanismen) auf die strategische Situation von Agenten vor der Entscheidung zur Netzwerkteilnahme angewendet.

Dabei bezeichnet „Kooperationsnetzwerk“ ein System von Geschäftspartnern, die durch Dependenzen wie insbesondere Netzeffekte verbunden sind und die hiermit einhergehenden Synergiepotenziale nutzen (möchten). „Standards“ bezeichnen Technologien oder Produkte, die aufgrund technischer Spezifikationen Kompatibilität schaffen und hierdurch Nutzernetzwerke konstituieren.

Nachfolgend werden zunächst die betrachteten Koordinationsprobleme systematisiert (3.1), am Beispiel Straight Through Processing im Wertpapierhandel diskutiert (3.2) und anschließend die für die weitere Untersuchung notwendigen Gleichgewichts- und Effizienzkonzepte für eine ökonomische Netzwerkanalyse entwickelt.

\subsection{Koordinationsprobleme in Geschäftsnetzwerken}

Neben unvollständiger Information über das Entscheidungsverhalten der Netzwerkpartner resultiert ein Koordinationsproblem im Kontext der Entscheidung der Netzwerkteilnahme aus der Asymmetrie der hiermit verbundenen Netzkosten und Netznutzen. Dies wird, neben einer vielfach beobachtbaren Unterschätzung der Netzpotenziale, häufig als Ursache einer zögerlichen Vernetzung (excess inertia, Start-up-Problem) gesehen (siehe ,,aggressives Abwarten“ in Abschnitt 2.1).

Abhängig von der institutionellen Einbettung der Netzakteure können Netzwerkteilnahmeentscheidungen prinzipiell zentral koordiniert sein oder dezentral, wie im Falle souveräner Geschäftseinheiten mit autonomen IT-Budgets [West ${ }^{+} 99$ ]. 
Während eine idealtypische Optimallösung für die Ermittlung der Netzwerkteilnehmer (,zentrale Koordination“) theoretisch ermittelt werden kann (siehe Abschnitt 4), sind reale Netzwerke häufig weniger effizient (,,dezentrale Koordination"). Aus einer theoretischen Sicht implizieren Netzeffekte - im Gegensatz zu den vorab beschriebenen ,viktorianischen“ Gleichgewichten der Neoklassik - multiple Gleichgewichte [Arth89]. Daher ist Netzwerkverhalten schwierig vorherzusagen und Planung, Kontrolle und Betrieb von Kooperationsnetzen stellen eine komplexe Herausforderung dar. In der Praxis zeigt sich dies als das Problem, ein möglichst vorteilhaftes Netzwerk als optimale Art und Menge der Verbindung von individuellen Netzakteuren (Individuen, Geschäftseinheiten etc.) aufzubauen. Prominente Beispiele sind EDI-Netze oder elektronische Marktplätze. Der Trade-off besteht dabei zwischen Netznutzen in Form von Kosteneinsparungen durch tiefere Integration mit den Systemen der Partner und reduzierte Friktionskosten einerseits und Netzwerkteilnahmekosten, etwa durch Systemanpassungen zur Herstellung von Kompatibilität (also Standardisierung) andererseits. Aus individueller Sicht ist Netzwerkteilnahme vorteilhaft, wenn die Nutzen die Kosten übersteigen. Allerdings emergieren zwischen völlig autonomen Agenten nicht immer vorteilhafte Lösungen. Viele Akteure verharren in ineffizienten Suboptima und substantielles Netzwerkpotenzial bleiben ungenutzt.

\subsection{Das Beispiel Straight Through Processing im Wertpapiergeschäft}

Ein aktuelles Beispiel für Koordinationsherausforderungen im E-Business ist Straight Through Processing (STP) für eine grenzüberschreitende End-to-EndIntegration im Wertpapierhandel [Weiz $\left.{ }^{+} 03\right]$, vergleichbar mit anderen Integrationsproblemen in EDI-Netzwerken der Automobilindustrie [ $\left.\mathrm{Fric}^{+} 02\right]$.

Insbesondere fehlende Kommunikationsstandards und mangelnde Kompatibilität zwischen der Vielzahl verwendeter Abwicklungssysteme (intern) sowie national unterschiedliche Anforderungen und Rahmenbedingungen (extern) stehen derzeit einer schnellen, automatisierten und grenzüberschreitenden Abwicklung von Wertpapiertransaktionen entgegen [Swif00, S.28]. Durch die Automatisierung der System-zu-System-Kommunikation und die damit einhergehende Vermeidung von Medienbrüchen erhoffen sich die Netzwerkpartner die Beschleunigung gemeinsamer Prozesse sowie eine Reduktion von Fehlern und Prozesskosten [Emme93, S.17-29]. 


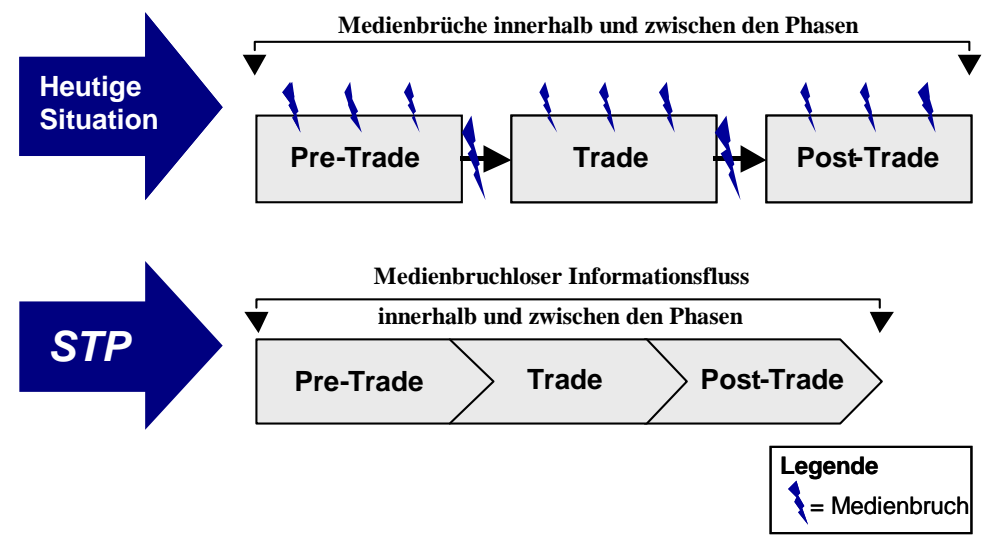

Abbildung 1: Konzept des Straight Through Processing

Grundsätzlich sind analoge Integrationsprobleme aus der Literatur bekannt, es lässt sich indes eine besonders ausgeprägte Rolle der Banken feststellen durch

- eine sehr starken Heterogenität der IT-Landschaften durch nationale und branchenspezifische Rahmenbedingungen und IT-Strategien,

- eine große Anzahl von 30 bis 40 unterschiedlichen Systemen innerhalb einer Bank, die einzelne Bankenprodukte unterstützen [Ruh ${ }^{+} 01$, S.78] und

- eine hinter dem Stand anderer Branchen zurückliegende Industrialisierung im Finanzsektor, mit einem hohen Anteil von 60\% an Eigenentwicklungen [Shah02].

Entsprechend häufige Medienbrüche führen u. a. dazu, dass 15-20\% aller grenzüberschreitenden Transaktionen in Wertpapieren mit Fehlern behaftet sind. Die Fehlerrate von Geschäften mit Schwellenländern (Emerging Markets) liegt bei bis zu 33\% [Gilk01]. Laut SWIFT macht allein der Kostenanteil für die Fehlerkorrektur etwa ein Drittel der gesamten Abwicklungskosten oder $€ 1$ Mrd. pro Jahr aus [Brow01]. Reuters schätzt das Einsparvolumen durch effiziente Handels- und Abwicklungsprozesse auf jährlich rund \$ $12 \mathrm{Mrd}$. [Reut01].

Aber wie auch die EDI-Historie zeigt, ist die Gestaltung dieser Netzwerke eine schwierige Herausforderung. Gerade im STP-Kontext zeigen sich die berüchtigten Start-up-Probleme: Da die erwarteten (internen und externen) STP-Nutzen hohe (interne) STP-Raten bei den Partnern voraussetzen, besteht ein Anreiz, deren Investition in interne und externe Schnittstellen und Systemintegration abzuwarten und damit das Risiko der frühen - und möglicherweise alleinigen und damit unrentablen - Investition zu vermeiden. Neben diesem Start-up-Problem [KaSh85; Rohl74; OrSm81] ist ein zweites Phänomen bedenkenswert. Da ein echtes STP die Partizipation aller Prozessbeteiligten verlangt und die zu Grunde liegenden Prozesse leicht Dutzende unabhängiger Partner in den unterschiedlichsten Regionen 
der Welt betreffen können [Emme93, S.6-8], kann die Situation entstehen, dass für einen bestimmten Akteur zwar aus individueller Sicht die Teilnahme nicht lohnenswert ist, dieser Akteur aber aus Sicht der gesamten Kette, oder einer Mehrheit der Partner, teilnehmen sollte. Genau diese Diskrepanz verdeutlicht ein grundlegendes E-Business-Problem, nämlich die Koordinationsherausforderung für Netzwerke als Synchronisation lokaler und globaler Effizienz. Die Bedeutung dieser beiden grundsätzlichen Probleme - Start-up-Problem und grundsätzliche Konflikte bezüglich der Teilnahmevorteilhaftigkeit - sind deren Implikation auf die Kosten möglicher Lösungsdesigns. Im ersten Fall kann das Start-up-Problem prinzipiell recht einfach etwa durch Informationsintermediation gelöst werden. Dies ist möglich, da Netzwerkteilnahme sowohl aus individueller als auch aggregierter Netzwerkperspektive wünschenswert ist. Die ist der Standardfall in den meisten neoklassischen Modellen. Durch die Existenz von Netzeffekten ist das zweite Problem schwieriger, da es regelmäßig teure Ausgleichsmechanismen wie Querzahlungen erfordert. Als Beispiel dieser Problematik im STP-Umfeld kann die abwartende Haltung vieler Banken gesehen werden, die vor der Entscheidung stehen, den Transaction-Flow-Manager (TFM), eine Softwarelösung der GSTPAInitiative für ein Straight Through Processing v. a. im Cross-Border-Geschäft mit Wertpapieren, zu verwenden. So gilt der TFM prinzipiell als hilfreich, wenn alle teilnehmen, allerdings wird die Kosten-Fairness auf Grund individuell unterschiedlich hoher Implementierungskosten sehr unterschiedlich gesehen [Weiz ${ }^{+} 03$ ].

\section{3 Ökonomische Netzwerkanalyse: Netzeffekte und Effizienz}

Die Effizienz von und in Netzwerken wird häufig in Form einer netzweit aggregierten Größe wie Prozessdurchlaufzeit über alle Elemente einer Wertkette oder netzweite Kosten operationalisiert (,zentrale“ Lösung). Demgegenüber beschreibt eine derartige gemeinsame Größe, die eine kollektive Nutzenfunktion impliziert, nicht die strategische Investitionssituation der individuellen Netzwerkagenten, welche eher eine individuell als kollektiv optimale Strategie anstreben (,dezentrale“ Lösung). Diese Diskrepanz ist (mit-) verantwortlich für die häufig weit hinter den Erwartungen und Möglichkeiten zurückbleibende Effizienz von Netzwerkinfrastrukturen (siehe Start-up-Problem). Im neoklassischen Modell gibt es keinen Unterschied zwischen diesen beiden Szenarien, wenn bestimmte Annahmen insbesondere bzgl. der individuellen Nutzenmaximierung sowie weiterer Eigenschaften des homo oeconomicus gelten und aufgezeigt wird, dass die Wohlfahrtstheoreme gelten und die Verfolgung der Individualziele der Agenten mit dem Kollektivziel der Ökonomie synchron läuft [HiKi76]. Eine zentrale Annahme hierbei ist die Nicht-Existenz von Externalitäten. Leider zerstören, wie eingangs beschrieben, Netzeffekte als Form von Externalitäten die automatischen Transmission von lokaler zu globaler Effizienz [Weit ${ }^{+} 00$ ]. Dabei liegt nach traditioneller Definition eine Externalität vor, wenn die Nutzenfunktion $U_{i}($.$) einiger Akteure i Variablen$ enthält, deren Wert durch einen anderen Agenten $\mathrm{j}$ bestimmt wird, der die Wohl- 
fahrtseffekte seiner Handlungen für das Nutzenniveau des i nicht berücksichtigt. Eine jüngere Definition besagt, dass eine Externalität existiert, wenn es nicht ausreichend Anreize zur Entstehung eines (potenziellen) Marktes für bestimmte Güter gibt und die Nicht-Existenz des Marktes zu nicht-pareto-effizienten Gleichgewichten führt [Weit03]. Im Einklang mit der klassischen ökonomischen Literatur existiert eine Netzwerkexternalität, wenn die Marktteilnehmer den Einfluss ihrer Netzwerkteilnahme auf andere nicht zu internalisieren vermögen. Für den gängigen Fall positiver Netzeffekte bedeutet dies, dass die Akteure am Wertzuwachs des Gesamtnetzes, der durch ihre Teilnahme entsteht, nicht partizipieren können. Bei positiven Netzexternalitäten ist damit der private Anreiz zur Netzwerkteilnahme kleiner als der soziale und es kommt daher zu nicht ausreichend großen Netzen. Es stellt sich die Frage der optimalen Internalisierung dieser Netzeffekte: Mit welchen Koordinationsmechanismen können gute Netzwerke entstehen?

\subsection{Gleichgewichtskonzepte in Netzwerken}

Bei Existenz mehrerer Gleichgewichte stellt sich die Frage, wie diese zu unterscheiden sind bezüglich Effizienz und Erreichbarkeit bzw. wie sie in eine Präferenzreihenfolge zu bringen sind. Welches Gleichgewicht kann, wird oder soll aus Sicht eines individuellen Netzteilnehmers, welches aus Sicht des Gesamtnetzes erreicht werden? Zu den in der Literatur diskutierten Kriterien zur Bewertung von Gleichgewichten [NeMo67, S.49-52] sind im Kontext der Gleichgewichtsanalyse in Netzwerken insbesondere Pareto-Effizienz und Kaldor-Hicks-Effizienz nützlich [Fees00, S.54-57].

Als zentrales mikroökonomische Konzept zur wohlfahrtstheoretischen Beurteilung von stabilen Gleichgewichten hat sich das Pareto-Effizienz-Kriterium etabliert. Ein Zustand heißt pareto-effizient, wenn es nicht möglich ist, ein Wirtschaftssubjekt besser zu stellen, ohne mindestens ein anderes Individuum schlechter zu stellen. Formal ist eine Allokation x pareto-effizient dann und nur dann, wenn keine Allokation y existiert, die von allen Individuen schwach und von mindestens einem stark präferiert wird. Das Konzept der Pareto-Effizienz bewahrt die Ordinalität der Nutzentheorie durch die Vermeidung interpersoneller Nutzenvergleiche, erkauft sich dies aber durch die Unmöglichkeit, verschiedene pareto-effiziente Gleichgewichte, die es in Netzen häufig gibt, vergleichen zu können. Das KaldorHicks-Kriterium beschreibt eine Präferenzordnung für verschiedene ParetoGleichgewichte [Fees00, S.56-67; BöI195, S.259-260]. Die Grundidee besteht in einer möglichen Kompensation zwischen allen Beteiligten, sodass letztlich alle besser gestellt sein könnten (oder zumindest nicht schlechter). Das Kriterium setzt nicht voraus, dass die Kompensation tatsächlich stattfindet (womit es identisch mit dem Pareto-Kriterium wäre). Ein Problem des Kaldor-Hicks-Konzeptes ist, dass es genau den intersubjektiven Nutzenvergleich voraussetzt, den das ParetoKonzept zu vermeiden sucht. 


\begin{tabular}{|c|c|c|}
\hline & \multicolumn{2}{|c|}{ Spieler 2} \\
\hline Spieler 1 & $\mathrm{~S}_{21}$ & $\mathrm{~S}_{22}$ \\
\hline $\mathrm{s}_{11}$ & $(3,4)$ & $(2,3)$ \\
\hline $\mathrm{s}_{12}$ & $(1,2)$ & $(5,3)$ \\
\hline
\end{tabular}

Tabelle 1: Auszahlungsmatrix für Netzwerkteilnahmespiel mit zwei pareto-effizienten (P) und einem kaldor-hicks-effizienten (K) Gleichgewicht

Das Spiel in Tabelle 1 weist zwei pareto-effiziente Nash-Gleichgewichte auf $\left(\left(\mathrm{s}_{11}, \mathrm{~s}_{21}\right) ;\left(\mathrm{s}_{12}, \mathrm{~s}_{22}\right)\right)$, wobei durch die Pareto-Effizienz keine Aussage über die Rangfolge der Gleichgewichte gemacht wird. Das Kaldor-Hicks-Kriterium besagt nun, dass Zustand $\left(\mathrm{s}_{12}, \mathrm{~s}_{22}\right)$ eindeutig vorzuziehen ist, da durch eine Kompensationszahlung von einer Einheit Spieler 2 den erlitten Verlust durch den Wechsel von $\left(\mathrm{s}_{11}, \mathrm{~s}_{21}\right)$ auf $\left(\mathrm{s}_{12}, \mathrm{~s}_{22}\right)$ wieder sein altes Niveau erreichen kann (Pfeil in Tabelle 1). Spieler 1 hätte trotz Kompensationszahlung immer noch ein höheres Ergebnis als im Gleichgewicht $\left(\mathrm{s}_{11}, \mathrm{~s}_{21}\right)$.

\subsection{Bedeutung der Gleichgewichtskonzepte für die Netzwerk- analyse}

Welche Netzwerkstrukturen entstehen nun unter zentraler und dezentraler Koordination und wie effizient sind sie? Zur Untersuchung der Effizienz der Gleichgewichte werden die beiden Konzepte der Pareto- und Kaldor-Hicks-Effizienz verwendet. Positive Netzeffekte lassen sich in Auszahlungs-(Bi-)Matrizen an den höheren Werten der Hauptdiagonale gegenüber der Nebendiagonale ablesen. Das typische Vernetzungsspiel ist ein „Battle of the Sexes“ [FaSa88, S.238], das seinen Namen von der strategischen Situation bezieht, dass ein Paar gemeinsam den Abend verbringen möchte, er dies aber beim Boxkampf und sie im Theater, wobei die gemeinsame Abendgestaltung wichtiger ist als die Veranstaltung. Historische Beispiele sind u. a. Fernsehstandards (PAL, SECAM, NTSC) [Cran79] oder Radio (AM stereo) [BeJo86].

Existenz und Effizienz der resultierenden Gleichgewichte in Netzwerken sind stark abhängig von der Spezifikation der strategischen Situation der Spieler wie insbesondere bezüglich der verfügbaren Informationen sowie der Entscheidungsstruktur (statisch bzw. einmalig vs. sequenziell vs. wiederholt). Als ein wichtiges Ergebnis zeigt sich, dass es bei dezentraler Koordination kein eindeutiges Gleichgewicht (in reinen Strategien) gibt: Entweder es gibt ein Glerichgewicht, nämlich die Nichtteilnahme, oder es gibt zwei Gleichgewichte (beide/keiner nimmt teil). Diese Grundaussage findet sich auch bei komplexeren Spielen [Weit03]: In dezen- 
tral koordinierten Netzen gibt es eine schwächere Teilnahmetendenz. Diese Abweichung von der zentralen Lösung wird nachfolgend Effizienzlücke (oder Netzwerklücke) genannt und findet sich in Abbildung 2 (siehe Abschnitt 4.3). Es wird auch deutlich, dass im Falle mehrerer Gleichgewichte die zentrale Lösung stets der Kaldor-Hicks-Lösung entspricht. Allgemein sind die meisten dezentralen Gleichgewichte pareto-effizient, die zentrale Lösungsgüte kann indes nicht immer erreicht werden. Insbesondere im Fall, dass ein Spieler Verluste aus Netzwerkteilnahme erleidet, insgesamt die Nutzen aber die Kosten übersteigen, sind die Ergebnisse dezentraler Koordination verbesserungsfähig, etwa durch die Vereinbarung von Kompensationszahlungen.

Damit ergeben sich zwei grundsätzliche Probleme: Entweder benötigen die Spieler in Situationen, in denen allseitige Vernetzung wünschenswert ist, in denen es aber multiple Gleichgewichte gibt, mehr Informationen, um den Teil der Netzwerklücke zu überwinden, der sich nur aus der Unsicherheit über das Verhalten der Partner ergibt. Oder es gibt Situationen, in denen eine Koordination der Vernetzungsentscheidungen mit Ausgleichszahlungen oder dergleichen einhergehen müsste.

Diese Unterscheidungen werden sich beim Vergleich unterschiedlicher Entscheidungsszenarios im Rahmen der Simulationen des nächsten Kapitels als zweckmäBig erweisen.

\section{Ein Netzwerkmodell}

Nach einer kurzen Erläuterung des verwendeten Simulationsmodells sowie der zu Grunde liegenden Parameter (4.1 und 4.2) wird die individuelle Effizienz zentraler und dezentraler Koordination untersucht und im anschließenden Kapitel 5 diskutiert.

\subsection{Ein einfaches Simulationsmodell}

Das nachfolgend vorgestellte Simulationsmodell basiert auf dem Modell von [Weit03] [West ${ }^{+}$99] [Buxm ${ }^{+}$99]. Zur Verwendung von Simulationsmodellen siehe Tesfatsion [2002]; Vriend [Vrie96; Vrie99], Weitzel und König [WeKö03]. Eine ausführlichere Modellierung des dezentralen Falls mit weiteren Ergebnissen zu Netzwerktopologien, Installed-Base-Effekten, multiplen Technologien etc. bietet [Weit03], eine ausführliche Herleitung des zentralen Modells [Buxm96].

Die Nutzen der Netzwerkteilnahme resultieren aus verbessertem Informationsaustausch zwischen Kommunikationspartnern [Klme98, S.63], schnellerer Kommunikation mit geringeren Friktionskosten [BrWh85; Thum95, S.14-15) und weiteren strategischen Vorteilen wie Just-in-Time-Produktion [Pico $\left.{ }^{+} 93\right]$. Die Kosten 
der Netzwerkteilnahme umfassen technische und organisatorische Integrationsaufwendungen (Hardware, Software, Training etc.).

Die Knoten i ( $\mathrm{i}=\{1, \ldots, \mathrm{n}\})$ eines Kommunikationsnetzwerkes sind die Kommunikationspartner (menschliche wie maschinelle Akteure). Die Netzkanten bilden die mit einsparbaren Kommunikationskosten bewerteten Kommunikationsbeziehungen ab. $K_{i}$ beschreiben die Kosten der Netzwerkteilnahme für Agent $i$ und $\mathrm{c}_{\mathrm{ij}}$ die Vorteile für Agent $\mathrm{i}$ aus der Netzteilnahme von Agent $\mathrm{j}$ (bzw. die hierdurch mögliche Vermeidung der (Friktions-)Kosten zu anderen Netzwerkpartnern j). Um individuelle und aggregierte Netznutzen vergleichen zu können, werden zwei grundsätzliche Koordinationsformen modelliert: In dezentral koordinierten Netzwerken wird individuelles Agentenverhalten im extremen Fall der Nichtexistenz von Ex-ante-Koordination oder externem Zwang beschrieben. Im Gegensatz hierzu beschreibt zentrale Koordination die idealtypische optimale Koordinationsgüte für das Gesamtnetz unter der Annahme, dass eine zentrale, allwissende Instanz eine netzweit optimale Lösung (etwa aus Sicht eines Netzbesitzers) bei Nichtexistenz von Agency- und Kontrollkosten ermitteln und auch durchsetzen kann. Damit sind die lokalen Auswirkungen aus zentraler Sicht irrelevant.

Die (dezentrale) Netzwerkteilnahmebedingung für Agent i lautet:

$$
\sum_{\substack{j=1 \\ j \neq i}}^{n} c_{i j}-K_{i}>0
$$

In Entscheidungssituationen autonomer Agenten ist es indes zweifelhaft, ob alle Partner teilnehmen (Netzeffekt), sodass i die Partizipationsentscheidung der Partner $\mathrm{j}(\mathrm{j} \in\{1, \ldots, \mathrm{n}\} ; \mathrm{i} \neq \mathrm{j})$ antizipieren kann als (ex ante)

$$
\begin{aligned}
& \operatorname{EXPECT}\left[E_{i}\right]=\sum_{\substack{j \neq 1 \\
j \neq i}}^{n} p_{i j} c_{i j}-K_{i}=\sum_{\substack{j=1 \\
j \neq i}}^{n} \frac{c_{j i}(n-1)-K_{j}}{c_{j i}(n-1)} c_{i j}-K_{i} \\
& \text { s.t. } \quad c_{j k}>0 \quad \forall k \in\{1, \ldots, n\}
\end{aligned}
$$

$p_{i j}$ beschreibt die Wahrscheinlichkeit, die Agent i der Teilnahme von $\mathrm{j}$ zumisst. Gilt EXPECT $\left[\mathrm{E}_{\mathrm{i}}\right]>0$, wird i Teil des Netzes. Siehe Buxmann et al. [Buxm ${ }^{+}$99] für eine ausführliche Beschreibung.

\subsection{Simulationsaufbau und Parameter}

Der individuelle Ex-post-Nutzen für Agent $\mathrm{i}$ aus Netzwerkteilnahme $\mathrm{E}_{\mathrm{i}}$ bemisst sich entsprechend Gleichung 3 (die Binärvariable $x_{i}$ nimmt bei Netzteilnahme den Wert 1 an): 


$$
E_{i}=\sum_{\substack{j=1 \\ j \neq i}}^{n} c_{i j} \cdot x_{j}-K_{i}
$$

Gemäß Gleichung 4 beschreibt CE die aggregierten, netzweiten Auswirkungen der Teilnahmeentscheidungen ("coordination efficiency") als horizontale Aggregation der individuellen Nutzen.

$$
C E=\sum_{i=1}^{n} E_{i}=\sum_{i=1}^{n} \sum_{\substack{j=1 \\ j \neq i}}^{n} c_{i j} \cdot\left(1-y_{i j}\right)-\sum_{i=1}^{n} K_{i} \cdot x_{i}=\sum_{i=1}^{n} \sum_{\substack{j=1 \\ j \neq i}}^{n} c_{i j}-\left(\sum_{i=1}^{n} K_{i} \cdot x_{i}+\sum_{i=1}^{n} \sum_{\substack{j=1 \\ j \neq i}}^{n} c_{i j} \cdot y_{i j}\right)
$$

Die Formulierung in Gleichung 4 erlaubt die Herleitung von CE aus Ex-ante- und Ex-post-Kosten. $y_{\mathrm{ij}}$ nimmt den Wert Null an, wenn $\mathrm{i}$ und $\mathrm{j}$ Netzmitglieder sind. Natürlich ist die Interpretation der aggregierten Nutzen CE nicht unproblematisch in dezentralen Netzwerken, da dort der Fokus auf Individualentscheidungen liegt. Ziel ist die Konstruktion einer Vergleichsgröße möglicher Entscheidungsgüte bei der Entscheidung über die Vernetzung von Akteuren. Der Rest dieses Beitrages konzentriert sich daher auf die individuellen Auswirkungen im Sinne unterschiedlicher Vorteilhaftigkeit zentraler und dezentraler Koordination für individuelle Netzwerkakteure.

Der verwendete Simulator wurde mit JAVA 1.3 entwickelt, die Datenauswertung erfolgte mit SPSS 10.0. Zunächst wurden zufällig erzeugte Netzwerke initialisiert und allen Agenten approximativ normalverteilte individuelle Kosten und Nutzenwerte zugeordnet (gemäß den unten angegebenen Verteilungsparametern).

$$
\begin{aligned}
& \mathrm{CE}=\sum_{i=1}^{n} \sum_{j=i+1}^{n}\left(c_{i j}+c_{j i}\right) \cdot\left(1-y_{i j}\right)-\sum_{i=1}^{n} K_{i} \cdot x_{i} \rightarrow \max ! \\
& \text { u.d.N.: } \\
& \mathrm{x}_{\mathrm{i}}+\mathrm{x}_{\mathrm{j}}+\mathrm{y}_{\mathrm{ij}} \leq 2 \\
& \forall i, j \in n ; i<j \\
& \mathrm{x}_{\mathrm{i}}+\mathrm{y}_{\mathrm{ij}}>1 \quad \mathrm{x}_{\mathrm{j}}+\mathrm{y}_{\mathrm{ij}} \geq \\
& \forall i, j \in n ; i<j \\
& \mathrm{y}_{\mathrm{ij}} \geq 0 \\
& \mathrm{y}_{\mathrm{ij}} \leq 1 \\
& \forall i, j \in n ; i<j \\
& \mathrm{x}_{\mathrm{i}} \geq 0 \\
& \mathrm{x}_{\mathrm{i}} \leq 1 \\
& \forall i \in n
\end{aligned}
$$

Auf dieser Grundlage entscheiden die Agenten entsprechend den oben entwickelten Funktionen über ihre individuelle Netzteilnahme über T Perioden hinweg, wobei sie in jeder Periode auf ihre beobachtbare Umwelt reagieren, insbesondere also auf Teilnahmeentscheidungen anderer, die die eigene Unsicherheit reduzieren. Parallel wird für jedes Netz eine zentrale Vergleichslösung gemäß Gleichung 5 bestimmt (die Formulierung des zentralen Modells ist eine Adaption aus [Buxm96], 
für die Lösung wurden die JAVA-Packages lp.solve 2.0 von M. Berkelaar verwendet (http://siesta.cs.wustl.edu/ javagrp/help/LinearProgramming.html)).

Dieser Simulationsprozess wird 50 mal wiederholt, bevor die erwarteten Teilnahmekosten, beginnend bei $\mu(K)=45.000$, jeweils um 250 reduziert werden und der Prozess neu gestartet wird. Die nachfolgende Abbildung besteht somit aus 4.500 Simulationsläufen.

\subsection{Simulationsergebnisse}

In Abbildung 2 zeigen die Boxen die individuellen Nutzen (0,25-0,75-Quantile, der Querstrich ist der Median, Antennen markieren alle $E_{\mathrm{i}}$ ) aus Netzwerkteilnahme $\left(\mathrm{E}_{\mathrm{i}}\right)$ bei unterschiedlichen Teilnahmekosten $\mathrm{K}_{\mathrm{i}}$ (.cen beschreibt zentrale und dec dezentrale Koordination).

Es zeigt sich die intuitive Diskrepanz zwischen zentraler und dezentraler Koordination als vertikaler Abstand der beiden Lösungen $\mathrm{E}(\mathrm{cen})$ und $\mathrm{E}(\mathrm{dec})$ bei unterschiedlichen $\mu(\mathrm{K})$, der das Ausmaß der internalisierbaren Netzeffekte quantifiziert (,Netzwerklücke“). Beispielsweise ist der Median die netzweite Verbesserung bei der zentralen Lösung (E(cen)) bei Teilnahmekosten von $\mu(K)=24.000$ ca. 10.000 , während bei dezentraler Koordination das Netz noch inaktiv ist $(\mathrm{E}(\mathrm{dec})=0)$. Offensichtlich ist in dezentralen Netzen mit vergleichsweise weniger Partizipation zu rechnen.

Um die Entwicklung von $E_{\mathrm{i}}$ im Zeitablauf zu verdeutlichen, sind für den Fall der dezentralen Koordination die Nutzen in $\mathrm{t}=1$ sowie im Gleichgewicht (stationärer Zustand $\mathrm{t}=\mathrm{T}$ ) abgebildet. Interessanterweise ergeben sich vergleichsweise wenig negative $\mathrm{E}_{\mathrm{i}}$, vor allem im Falle zentraler Koordination. Dies bedeutet, dass der Fall der „schwierigen“ Gleichgewichte, in denen Agenten durch Netzwerkteilnahme individuell Verluste erleiden, aus zentraler Sicht aber nichtsdestoweniger teilnehmen sollten (oder die im dezentralen Kontext Subventionen verlangen würden), insgesamt eher selten ist. Es zeigt sich, dass nicht nur das Gesamtnetz, sondern auch eine substanzielle Mehrheit der Individuen durch zentrale Koordination besser gestellt wären. Der Anteil dieser Individuen ist natürlich stark abhängig von der Streuung der Kosten und Netzeffekte und wird, auch im Kontext der Wahl zwischen unterschiedlichen Netzwerken, in [Weit ${ }^{+} 03$ ] ausführlich untersucht.

Diese Erkenntnisse und Systematisierungen erweisen sich als nützlich bei der Überlegung nach geeigneten Koordinationswegen zur Schließung der Netzwerklücke: Wenn kein $\mathrm{E}_{\mathrm{i}}(\mathrm{cen})<0$, dann ist die zentrale Lösung pareto-superior zur dezentralen. Dies bedeutet, dass alle Netzwerkteilnehmer hiervon profitieren und es im Nachhinein selbst dann nicht bereuen, wenn sie gezwungen worden wären. Diese strategische Situation, die gelegentlich auch als Win-Win-Situation bezeichnet wird, ist prinzipiell einfacher lösbar als beispielsweise Konstantsummenspiele oder Situationen mit $\mathrm{E}_{\mathrm{i}}(\mathrm{cen})<0$. 


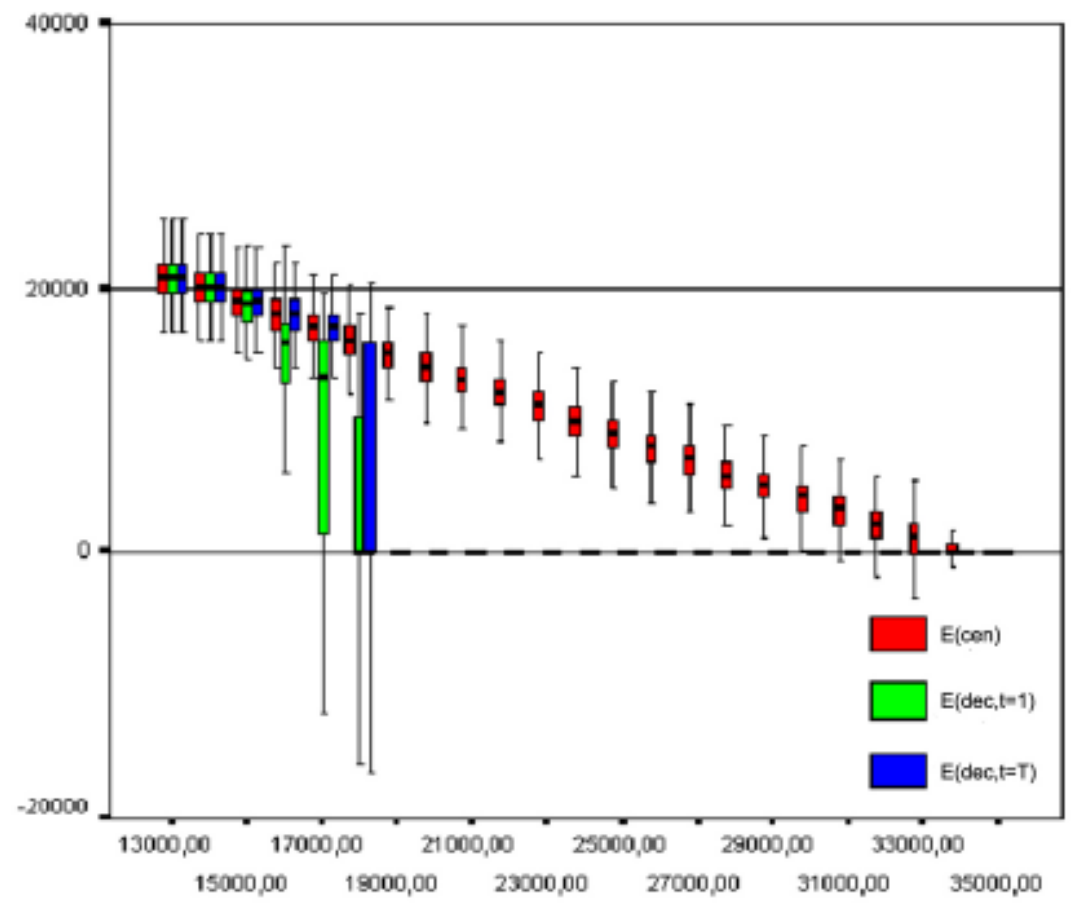

Abbildung 2: Individuelle Entscheidungsqualität $\mathrm{E}_{\mathrm{i}}$ (zentrale und dezentrale Koordination, Ordinate) bei unterschiedlichen Teilnahmekosten $\mu(\mathrm{K})$ (Abszisse)

\section{Diskussion der Ergebnisse}

\subsection{Allgemeine Lösungsansätze}

Was bedeuten die Simulationsergebnisse für die Frage nach möglichen Lösungsansätzen für Netzwerkprobleme und was sind die Implikationen für die Vernetzung von E-Business-Partnern? Es konnten zwei grundsätzliche Kosten-NutzenKonstellationen identifiziert werden. Entweder kann eine Ineffizienz durch falsche Antizipation der Partnerentscheidungen erklärt werden oder es gibt Agenten, die sich zwar aus zentraler Sicht vernetzen sollten, aus dezentraler (individueller) Perspektive allerdings hieraus Nachteile erleiden. Während der zweite Fall eine Redistribution von Teilen der „Netzgewinne“ verlangt, reicht im ersten Fall prinzi- 
piell eine Unsicherheitsreduktion, also Verbesserung der Informationsqualität der Entscheider bezüglich der Partnerentscheidungen, aus. Hier bieten sich grundsätzlich Versicherungslösungen für die beschriebenen Netzwerkprobleme an, die den Agenten exogen durchsetzbar zusichern, alle nach Abzug der Netznutzen verbleibenden Kosten zu übernehmen. Sollte der Ausgeber dieser Option (etwa ein Netzbesitzer) zu recht davon überzeugt sein, dass ein Gleichgewicht des ersten Falls vorliegt, ist dies ein eleganter (und dann auch kostenloser) Weg zur Lösung des Start-up-Problems. Dies setzt natürlich die Kenntnis der wünschenswerten Netzteilnehmer, also des Optimalnetzes, voraus. Weiterhin ergeben sich Probleme (und damit Kosten) der objektiven Ermittlung der zu Grunde liegenden Kosten und Nutzen. Bei den einfachen Gleichgewichten der ersten Art reicht also prinzipiell jede Art der Informationsintermediation, welche die Ex-ante- und die Ex-postEntscheidungen aneinander koppelt, ohne dass Querzahlungsmöglichkeiten notwendig sind. So könnte z.B. ein schwarzes Brett mit verbindlichen Ankündigungen oder ein „runder Tisch“ einen Bandwagon-Prozess starten [FaSa86]. Darüber hinaus erfordern Gleichgewichte der zweiten Art etwa einen Kompensationsplan, da die Option alleine hier nicht mehr ausreichen würde, die Lücke zu schließen.

Weiterhin darf eine Vielzahl von Engpässen bei der Modellierung nicht übersehen werden. So berücksichtigt das Modell derzeit ausschließlich positive Netzeffekte. Auch wenn ein Großteil der Literatur auf die Modellierung negativer Netzeffekte verzichtet, dürften diese den Anteil der Win-Win-Gleichgewichte, also der netztypisch „einfachen“ Koordinationsprobleme, deutlich beeinflussen. Neben SnobEffekten könnten zumal im E-Business-Kontext Individualitätsverluste und damit einhergehende Verschlechterungen der Wettbewerbsposition als negative Netzeffekte verstanden werden [Thum95, S. 26].

Es wurde diskutiert, dass Agenten an einem Netzwerk partizipieren, wenn sie dadurch einen Vorteil erlangen. Aber aufgrund von Informationsasymmetrien in dezentralen Netzwerken können einige Agenten unerwartet Verluste erleiden, da sich ihr Umfeld „falsch“ entschieden hat. Ein generelles Allokationsmodell zur Lösung dieses Problems ist insofern vorstellbar, als jedem Agenten die Wahl zwischen seinem Status Quo Ante und der Konstellation im stationären Zustand (also nach der Netzwerkkonstitution) eröffnet wird. Die endgültige Allokation wird dann und nur dann implementiert, wenn alle Agenten sie (zumindest schwach) präferieren [Vari94]. Wenn einige Agenten nicht wechseln wollen, könnte eine nächste Entscheidungsrunde ohne diese vorgenommen werden.

\subsection{Industriekonsortien als Lösungskonzept}

Ein Hauptergebnis vieler jüngerer Arbeiten zu Netzwerkeffizienz ist die Betonung der individuellen Netzwerknachbarschaft der Agenten (Netzwerktopologie). In diesem Kontext erscheinen Konsortialstrukturen als wichtige Koordinationskonzepte für Netzwerke, welche die beschriebenen Asymmetrien reduzieren helfen 
können. Entsprechend wird in vielen Beiträgen zu Standardisierungsstrategien und zwischenbetrieblichen Integrationsproblemen das Entwickeln gemeinsamer Strategien in Konsortien als Vorschlag zum Schließen der Netzwerklücke vorgebracht. In der Tat gibt es eine kaum überschaubare Fülle von Organisationen für Standards und Netzwerken. In letzter Zeit scheinen Industriekonsortien wie das W3C oder OASIS im Gegensatz zu staatlichen schnellere und bessere Ergebnisse zu entwickeln. Wenn tatsächlich bestimmte High-Tech-Branchen raschere (technologischer Entwicklungszyklus) und wegen der Netzeffekte mehr Koordination als traditionellere Branchen verlangen, stellt sich die Frage nach der geeigneten Koordinationsform. Die Simulationen im letzten Kapitel zeigen einen deutlichen Koordinationsbedarf z. B. zur Überwindung von Start-up-Problemen. Weiterhin konnte die Gleichgewichtsanalyse systematisieren, in welchen Fällen Koordination auf Grund informationeller Abmachungen anstelle von Querzahlungen möglich ist. Genau hier erscheinen Konsortien als attraktiver Lösungsansatz. Eine Hypothese, warum Industriekonsortien staatlichen Ansätzen vorzuziehen sind, liegt neben der Tatsache, dass sie für schneller gehalten werden, darin begründet, dass sie weniger verpflichtend sind: Farrell und Saloner zeigen in ihrem Modell von 1988 drei unterschiedliche Koordinationsprozesse, um sich auf einen gemeinsamen Standard zu einigen:

1. Staatliches Komitee mit Aktionspflichten für die Netzwerkteilnehmer: Wenn sich die Agenten fortwährend treffen, um gemeinsam vorteilhafte (bindende) Handlungen zu beschließen, befinden sie sich in jeder Periode in einer „Battle of the sexes"-Situation (siehe Abschnitt 3.5).

2. Markt (,bandwagon“): Die (dezentrale) Marktlösung ist modelliert als „Grabthe-dollar"-Spiel (wer zuerst zugreift, gewinnt den Dollar, doch wenn beide gleichzeitig zugreifen, verlieren beide).

3. Hybridform: Agenten können trotz Komiteeteilnahme auch individuell entscheiden.

Farrell und Saloner zeigen, dass das Komitee den Markt dominiert, allerdings durch das Hybridmodell selbst dominiert wird. "In a committee...nothing is likely to happen for a long time" [FarSa88, S.239], allerdings produziert rein dezentrale (Markt-)Koordination häufig ineffiziente Oligopole. Die Hybridlösung verbindet die Vorteile beider Ansätze.

\section{Zusammenfassung und Ausblick}

Die für das E-Business grundlegende Entscheidungen von Agenten zur Vernetzung wurde im Rahmen einer Gleichgewichtsanalyse modelliert. Auf Grund der aus der Existenz von Netzeffekten resultierenden Komplexität ist eine computergestützte Simulation verwendet worden, um Einsichten in die strategische Ent- 
scheidungssituation der Agenten und die Existenz und Effizienz zu erwartender Gleichgewichte zu gewinnen. Insbesondere das berüchtigte Start-up-Problem ließ sich gut erklären. Ein Blick auf die individuellen Konsequenzen der Netzwerkteilnahmeentscheidung zeigte, dass die strategische Entscheidungsproblematik der Agenten in dezentral koordinierten Netzen vielversprechend bezüglich einfacher Lösungsstrategien ist, da in vielen Fällen die Netzeffizienz durch einfache Informationsintermediation und ohne aufwändige Kostenverteilungen geschehen kann. Letztlich lässt die Existenz von Externalitäten genau dies vermuten: Die Auswirkungen einer Entscheidung auf andere werden nicht vollständig berücksichtigt. Wie in einem neoklassischen Markt mit Externalitäten ist ein Preis zu niedrig dimensioniert, um alle notwendigen Informationen zu tragen.

Die vorgestellten Ergebnisse können natürlich nur ein kleiner Beitrag zum besseren Verstehen der komplexen Dynamiken in Netzwerken sein. Wichtige Erweiterungen umfassen die Verwendung von Erkenntnissen aus anderen Disziplinen wie z. B. der Soziologie oder Geografie bezüglich Netzwerktopologie und Dichte. Zu Anforderungen an eine interdisziplinäre Netzwerktheorie siehe Weitzel et al. [Weit $\left.{ }^{+} 00\right]$. Im Kontext der Lösungsstrategien für Kooperationsnetzwerke und der vorgestellten Klassifikation von Kosten, Nutzen und der resultierenden strategischen Implikationen erscheint es lohnenswert, Internalisierungsstrategien für Netzwerke auf der Grundlage wichtiger Ergebnisse der Spieltheorie unter Berücksichtigung von Netzeffekten zu entwickeln und zu evaluieren. Ein Ansatz könnte auf lokale Koalitionen aufbauen, in denen Agenten mit ihren besten (z. B. größten $\mathrm{c}_{\mathrm{ij}}$ ) ein, zwei oder mehreren Partnern verbindliche „Minimalkonsortien“ bilden, die intern quasi zentral koordiniert sind. Modellerweiterungen in Weitzel et al. [Weit $\left.{ }^{+} 03\right]$ zeigen beispielsweise, dass eine „Konsortialtreppe“ in Form des Zusammenschlusses vergleichsweise wenig heterogener Partner (z. B. gleiche Supply-Chain-Ebene) mit nachfolgender, einfacherer Koordination dieser Cluster erfolgversprechend sein könnte. Bei diesem Ansatz wird die Anzahl notwendiger Kommunikationsakte drastisch reduziert [ShVa98, S.184], während gleichzeitig die Koordination zwischen im Durchschnitt homogeneren Agenten stattfindet und damit einfacher ist. Wenn die realen Koordinationskosten positiv von der Anzahl der zu koordinierenden Partner abhängt, können so eine optimale Anzahl von Internalisierungspartnern je Netzwerk-Tier gefunden und möglicherweise Hinweise auf die optimale Netzwerkgröße abgeleitet werden. Empirische Daten zur Koordinationskostenentwicklung im Vergleich zu den lokalen Teilnahmekosten könnten hier wertvolle Hilfestellung leisten. Mögliche Anwendungsdomänen zur Erhebung derartiger Daten sind EDI-Netzwerke, betriebliche Intranets und auch die STPCommunity. 


\section{Literatur}

[Agre98] Agre, P.: A graduate seminar on the economic, legal, and strategic aspects of technical compatibility standards, http://dlis.gseis.ucla.edu/people/pagre/ standards.html, 1998.

[Arth83] Arthur, W.B.: Competing technologies and lock-in by historical small events: the dynamics of allocation under increasing returns; International Institute for Applied Systems Analysis Paper WP-83-92, Laxenburg, Austria (Center for Economic Policy research, paper 43, Stanford), 1983.

[Arth89] Arthur, W.B.: Competing technologies, increasing returns, and lock-in by historical events, in: The Economic Journal, 99, March 1989, 116-131.

[Arth96] Arthur, W.B.: Increasing returns and the new World of business, in: Harvard Business Review, 74, July-August 1996, 100-109.

[BeFa94] Besen, S.M.; Farrell, J.: Choosing How to Compete: Strategies and Tactics in Standardization, in: Journal of Economic Perspectives, vol. 8, no. 2, 1994, 117-131.

[BeJo86] Besen, S.M.; Johnson, L.L.: Compatibility Standards, Competition, and Innovation in the Broadcasting Industry, in: RAND Corporation R-3453-NSF, November 1986.

[BöI195] Böventer, E.v.; Illing, G.: Einführung in die Mikroökonomie, 8. Auflage, München; Wien 1995.

[BrKa95] Branscomb, L.M.; Kahin, B.: Standards processes and objectives for the National Information Infrastructure, in: Kahin, B./ Abbate, J. (eds.): Standards Policy for Information Infrastructure, Cambridge: MIT Press, 1995.

[Brow01] Brown, J. (2001): Twists and turns on the straight-through route, in: EuroMoney, 384, April 2001, S. 112-118.

[BrWh85] Braunstein, Y.M.; White, L.J.: Setting technical compatibility standards: An economic analysis, in: Antitrust Bulletin, vol. 30, 1985, 337-355.

[Bund98] Bundesumweltministerium: Bundesumweltamt (Hrsg.): So geht's auch! Gasantrieb, Bonn 1998.

[Buxm+99] Buxmann, P.; Weitzel, T.; König, W.: Auswirkung alternativer Koordinationsmechanismen auf die Auswahl von Kommunikationsstandards, in: ZfB (Zeitschrift für Betriebswirtschaft), Ergänzungsheft 02/99 Innovation und Absatz, 1999, 133-151.

[Buxm96] Buxmann, P.: Standardisierung betrieblicher Informationssysteme, Wiesbaden 1996.

[CeKa82] Ceci, S.J.; Kain, E.L.: Jumping on the bandwagon: The impact of attitude polls on polling behaviour, Public Opinion Quarterly, 46, 1982, 228-242.

[Cowa90] Cowan, R.: Nuclear Power Reactors: A study in technological lock-in, in: The Journal of Economic History, vol. L, no. 3, Sept. 1990, 541-567.

[Cran79] Crane, R.: The Politics of International Standards, Norwood, N.J., 1979. 
[DaGr90] David, P.A.; Greenstein, S.: The economics of compatibility standards: An introduction to recent research, in: Economics of innovation and new technology, 1, 3-41, 1990.

[Davi85] David, P.A.: Clio and the economics of QWERTY, in: American Economic Review, Papers and Proceedings, vol. 75, 1985, 332-337.

[EcHi95] Economides, N.; Himmelberg, C.: Critical Mass and Network Size with Application to the US FAX Market, Discussion Paper EC-95-11, Stern School of Business, New York University, 1995.

[Econ00] Economides, N.: An interactive bibliography on the economics of networks and related subjects, http://www.stern.nyu.edu/networks/biblio.html, 2000.

[Emme93] Emmelhainz, M.A.: EDI: A Total Management Guide, 2nd edition, New York 1993.

[FaSa85] Farrell, J.; Saloner, G.: Standardization, Compatibility, and Innovation, in: Rand Journal of Economics 16, 1985, 70-83.

[FaSa86] Farrell, J.; Saloner, G.: Installed Base and Compatibility: Innovation, Product Preannouncements, and Predation, in: The American Economic Review, vol. 76, no. 5, December 1986, 940-955.

[FaSa87] Farrell, J.; Saloner, G.: Competition, compatibility, and standards: The economics of horses, penguins, and lemmings, in: Gabel H. Landis (ed.): Product standardization and competitive strategy, Amsterdam 1987, 1-21.

[FaSa88] Farrell, J.; Saloner, G.: Coordination through committees and markets, in: RAND Journal of Economics, Vol. 19, No. 2, Summer 1988, 235-252.

[Fees00] Feess, E.: Mikroökonomie - Eine spieltheoretisch- und anwendungsorientierte Einführung, 2. Auflage, Marburg 2000.

[Fric+02] Fricke, M.; Weitzel. T.; König, W.; Lampe, R.: EDI and Business-to-Business Systems: The Status Quo and the Future of Business Relations in the European Automotive Industry, in: Proceedings of the Sixth Pacific Asia Conference on Information Systems (PACIS-2002), http://much-magic.wiwi.uni-frankfurt.de/ tweitzel/paper/EDIautomotive.pdf, 2002.

[Gabe87] Gabel, H.L.: Product Standardization and Competitive Strategy, Amsterdam 1987.

[Gabe91] Gabel, H.L.: Competitive Strategies for Product Standards, London 1991.

[Gand94] Gandal, N.: Hedonic price indexes for spreadsheets and empirical test for network-externalities, in: Rand Journal of Economics, vol. 25, no. 1, 1994, 160-170.

[GeHö95] Gerwin, J.; Höcherl, I.: Video 2000: Strategische Produktpolitik bei internationalem Wettbewerb, in: Brockhoff, Klaus (Hrsg.): Management von Innovationen: Planung und Durchsetzung; Erfolge und Misserfolge, Wiesbaden 1995, 17-44, 217-244.

[Gilk01] Gilks, D.: The GSTPA Is Primed For Pilot. The European Union Banking \& Finance News Network (EUBFN): http://www.eubfn.com/arts/gstpa2.htm, 2001. 
[Gröh99] Gröhn, A.: Netzeffekte und Wettbewerbspolitik. Eine ökonomische Analyse des Softwaremarktes, Kieler Studien 296, Tübingen, 1999.

[Habe89] Habermeier, K.F.: Competing technologies, the learning curve, and rational expectations, in: European Economic Review 33, 1989, 1293-1311.

[HaTe90] Hartmann, R.S.; Teece, D.J.: Product emulation strategies in the presence of reputation effects and network externalities: some evidence from the minicomputer industry, in: Economics of innovation and new technology, vol. 1-2, 1990, 157-182.

[Heme75] Hemenway, D.: Industry wide voluntary product standards, Massachusetts 1975.

[HiKi76] Hildenbrand, W.; Kirman, A.P.: Introduction to equilibrium analysis, NorthHolland, Amsterdam, 1976.

[KaSh85] Katz, M. L.; Shapiro, C.: Network externalities, competition, and compatibility, in: The American Economic Review, vol. 75, no. 3, June 1985, 424-440.

[KaSh86] Katz, M. L.; Shapiro, C.: Technology adoption in the presence of network externalities. in: Journal of Political Economy, vol. 94, no. 4, 1986, 822-841.

[KaSh92] Katz, M. L.; Shapiro, C.: Product Introduction with Network Externalities, in: Journal of Industrial Economics, 40 (1), 1992, 55-83.

[KaSh94] Katz, M. L.; Shapiro, C.: Systems Competition and Network Effects, Journal of Economic Perspectives, 8, Spring 1994, 93-115.

[Kind83] Kindleberger, C. P.: Standards as Public, Collective and Private Goods. Kyklos International Review for Social Sciences, 36(3), 1983, 377-396.

[Klei93] Kleinaltenkamp, M.: Standardisierung und Marktprozeß - Entwicklungen und Auswirkungen im CIM-Bereich, Wiesbaden 1993.

[Klme98] Kleinemeyer, J.: Standardisierung zwischen Kooperation und Wettbewerb, Frankfurt 1998.

[Leib50] Leibenstein, H.: Bandwagon, snob, and Veblen effects in the theory of consumers demand, in: Quarterly Journal of Economics, 64 (2), 1950, 183-207.

[LiMa90] Liebowitz, S.J.; Margolis, S.E.: The fable of the keys, in: Journal of Law and Economics, vol. 33, 1990, 1 - 25.

[LiMa94] Liebowitz, S.J.; Margolis, S.E.: Network Externality: An Uncommon Tragedy, in: The Journal of Economic Perspectives, Spring 1994, 133-150.

[LiMa95a] Liebowitz, S.J.; Margolis, S.E.: Are Network Externalities A New Source of Market Failure?, in: Research in Law and Economics, 1995.

[LiMa95b] Liebowitz, S.J.; Margolis, S.E.: Path Dependence, Lock-In, and History, in: Journal of Law, Economics and Organization, 11, April 1995, 205-226.

[Maub+00] Mauboussin, M.J.; Schay, A.; Kawaja, S.G. (2000): Network to Net Worth, The Rising Role of the Replacement Cycle, Credit Suisse First Boston, Equity Research, Frontiers of Finance, vol. 5 (May 11, 2000), http://www.capatcolumbia.com/Articles/ FoStrategy/Ni1924.pdf, 2000. 
[Moch95] Moch, D.: Ein hedonischer Preisindex für PC-Datenbanksoftware: Eine empirische Untersuchung, in: Harhoff, D./Müller, M. (Hrsg.): Preismessung und technischer Fortschritt, Baden Baden, 1995.

[NeMo67] Neumann, J.v.; Morgenstern, O.: Spieltheorie und wirtschaftliches Verhalten (Theory of Games and Economic Behavior). Deutsche Übersetzung der 3. Auflage von M. Leppig, Würzburg 1967.

[Nigg94] Niggl, J.: Die Entstehung von Electronic Data Interchange Standards, Wiesbaden 1994.

[OrSm81] Oren, S.S.; Smith, S.A.: Critical Mass and Tariff Structure in Electronic Communications Markets, Bell Journal of Economics, 12, Autumn 1981, 467-87.

[Pico+93] Picot, A.; Neuburger, R.; Niggl, J.: Electronic Data Interchange (EDI) und Lean Management, in: Zeitschrift für Führung und Organisation, Nr. 1/1993, S. 20-25.

[Plum37] Plummer, A.: New British Industries in the Twentieth Century, London 1937.

[Reut01] Reuters: o.V., in: Venture Financial Systems Group: Straight Through Processing - Just in Time, www.venturefsg.com/article2.htm, 2001.

[Rohl74] Rohlfs, J.: A theory of interdependent demand for a communications service, in: Bell Journal of Economics 5(1), 1974, 16-37.

[Ruh+01] Ruh, W.A.; Maginnis, F.; Brown, W.: Enterprise Application Integration: a Wiley tech brief, New York 2001.

[Schi00] Schinzer, H.: Electronic Commerce, in: Mertens et al. (Hrsg.): Lexikon der Wirtschaftsinformatik, 3., vollständig neu bearbeitete und erweiterte Auflage, 2000.

[Shah02] Shahrawat, D.: EAI's Impact on Financial Institutions - An Interview With Dushyant Shahrawat. In: EAI Journal, http://www.eaijournal.com/PDF/Shahrawat.pdf, Feb. 2002.

[ShVa98] Shapiro, C.; Varian, H. R.: Information rules: A strategic guide to network economy, Boston, Massachusetts, 1998.

[SWIF00] Swift Solutions - July 2000, Nr.7, Informationsbroschüre, www.swift.com/ index.cfm?item_id=3488, 2000.

[Tesf03] Tesfatsion, L.: Agent- based computational economics, http://www.econ.iastate.edu/tesfatsi/ace.htm, 2003.

[Thum95] Thum, M.: Netzwerkeffekte, Standardisierung und staatlicher Regulierungsbedarf, Tübingen 1995.

[Vari94] Varian, H.: A Solution to the Problem of Externalities When Agents are WellInformed, in: American Economic Review, December 1994, 1278-93, also: Microeconomics/Economics Working Paper Archive at WUSTL, RePEc:wpa:wuwpmi:9401003.

[Vrie96] Vriend, N.: Rational Behavior and Economic Theory, Journal of Economic Behavior and Organization 29, 1996, 263-285.

[Vrie99] Vriend, N.: Was Hayek an ACE?, Working Paper 403, Queen Mary and Westfield College, University of London, UK, May 1999. 
[WaWa83] Warren, M.E.; Warren, M.: Baltimore - when she was what she used to be, 1850-1930, Baltimore: John Hopkins University Press, 1983.

[Weit+00] Weitzel, T.; Wendt, O.; Westarp, F.v.: Reconsidering Network Effect Theory, in: Proceedings of the 8th European Conference on Information Systems (ECIS 2000), http://www.wiwi.uni-frankfurt.de/ westarp/publ/recon/reconsidering.pdf, 2000.

[Weit+01] Weitzel, T.; Harder, T.; Buxmann, P.: Electronic Business und EDI mit XML, Heidelberg 2001.

[Weit+03] Weitzel, T.; Beimborn, D.; König, W.: Coordination In Networks: An Economic Equilibrium Analysis, forthcoming in: Information Systems and e-Business Management (ISeB), Springer 2003.

[Weit03] Weitzel, T.: Economics of Standards in Information Networks, Springer/Physica, New York, Heidelberg 2003.

[Weiz+03] Weitzel, T.; Martin, S.; König, W.: Straight Through Processing auf XML-Basis im Wertpapiergeschäft, in: Wirtschaftsinformatik 4/2003.

[WeKö03] Weitzel, T.; König, W.: Standardisierungstheorie und Netzeffekte: Agent-based Computational Economics als wirtschaftsinformatischer Beitrag zu einer interdisziplinären Netzwerktheorie, erscheint in: WIRTSCHAFTSINFORMATIK 5/2003.

[WeSK01] Weitzel, T.; Son, S.; König, W.: Infrastrukturentscheidungen in vernetzten Unternehmen: Eine Wirtschaftlichkeitsanalyse am Beispiel von X.500 Directory Services, in: WIRTSCHAFTSINFORMATIK 4/2001, 371-381.

[West+99] Westarp, F.v.; Weitzel, T.; Buxmann, P.; König, W.: The Status Quo and the Future of EDI, in: Proceedings of the 1999 European Conference on Information Systems (ECIS'99), 1999.

[West03] Westarp, F.v.: Modeling Software Markets - Empirical Analysis, Network Simulations, and Marketing Implications, Physica, Heidelberg, 2003.

[WeWe02] Weitzel, T.; Westarp, F.v.: From QWERTY to nuclear power reactors: Historic battles for the standard, in: Geis; Koenig; Westarp (Hrsg.): Networks - Standardization, Infrastructure, and Applications, Berlin, New York (Springer) 2002, 33-61.

[Wies90] Wiese, H.: Netzeffekte und Kompatibilität, Stuttgart 1990.

[Yang97] Yang, Y.: Essays on network effects, Dissertation, Department of Economics, Utah State University, Logan, Utah, 1997. 